\title{
Beyond CLABSI and CAUTI: broadening our vision of patient safety
}

\author{
Kaveh G Shojania
}

Department of Medicine and the Centre for Quality Improvement and Patient Safety, University of Toronto, Toronto, ON M4N 3M5, Canada

\section{Correspondence to} Dr Kaveh G Shojania, Sunnybrook Health Sciences Centre, Room H468, 2075 Bayview Avenue, Toronto, ON M4N 3M5, Canada;

kaveh.shojania@sunnybrook.ca

Accepted 19 February 2020 Published Online First 28 February 2020

\section{Linked}

- http://dx.doi.org/10.1136/ bmjqs-2019-009330

\section{Check for updates}

(c) Author(s) (or their employer(s)) 2020. No commercial re-use. See rights and permissions. Published by BMJ.

To cite: Shojania KG BMJ Qual Saf

2020;29:361-364.
In this issue of BMJ Quality \& Safety, Meddings $\mathrm{et} \mathrm{al}^{1}$ report the evaluation of a national effort to reduce two well-known safety targets, central line-associated bloodstream infection (CLABSI) and catheter-associated urinary tract infection (CAUTI). The paper's introduction helpfully informs readers of the context. Prior projects funded by the US Agency for Healthcare Research and Quality (AHRQ) have reported well-known successes for both these targets. ${ }^{2}{ }^{3}$ One national collaborative reported a greater than $40 \%$ reduction in CLABSI in intensive care units (ICUs). ${ }^{2}$ And, a comparably large project reported a $32 \%$ reduction in CAUTI in clinical units other than ICUs, but with no reduction occurring in ICUs. ${ }^{3}$

This lack of improvement for CAUTI in ICUs might perplex those familiar with the history of these interventions. The AHRQ On the CUSP: Stop CAUTI project $^{3}$ included the Comprehensive Unit-based Safety Program (CUSP) to support behavioural and cultural changes seen as crucial to support uptake of the technical elements of the CLABSI bundle ${ }^{4}$ and other checklist-type interventions. ${ }^{5}$ Why would an intervention for CAUTI modelled after one which has apparently worked so well for CLABSI in ICUs ${ }^{2} 6$ work only outside ICUs? This unexpected result, along with the fact that, even in the seemingly more successful CLABSI project $^{2}$ a substantial proportion of ICUs did not improve, led to the national collaborative now reported by Meddings et al. ${ }^{1}$

The programme recruited 366 ICUs from 220 US hospitals, with 274 ICUs providing complete data. Neither target showed significant improvements. For CLABSI, the incidencerate ratio (IRR) was 0.75 , but the $95 \%$ CI extended up to an increase of $1.08(p=0.13)$. CAUTI showed a similar result: $I R R=0.79$ but with a CI extending up to $1.06 .{ }^{1}$ Moreover utilisation for both catheters decreased only marginally and non-significantly.

The authors offer two possible explanations for these disappointing results. First, the participating sites showed suboptimal engagement. About $60 \%$ of ICUs viewed none of the online educational modules for CLABSI and $45 \%$ viewed none for CAUTI. The authors suggest this suboptimal engagement reflected a decreased focus on the socioadaptive elements ${ }^{578}$ of the CUSP model compared with past programme. ${ }^{2}{ }^{3}$ That said, reductions in CAUTI and CLABSI in these previous programmes showed no associations with improvements in unit culture. ${ }^{9}$ Unless CUSP produces improvements not detected by measures of culture, the decreased focus on CUSP seems unlikely to explain the current null results.

A second possible explanation involves a ceiling effect from secular trends. ICUs in this collaborative started with average rates of both CLABSI and CAUTI below those seen following interventions in past successful programmes. $^{2}{ }^{3}$ With preintervention performance in the current programme already surpassing that seen with past successes, it does seem reasonable to suggest that secular trends in rates of CLABSI and CAUTI left little room for further reductions.

Though not suggested by the authors, a third possibility is simply that the technical intervention did not work. In the case of CLABSI, despite the prominence of the bundle, evidence for effectiveness remains mixed. ${ }^{10}$ Losses to follow-up of about $40 \%$ of eligible ICU months in the original study ${ }^{6}$ almost certainly produced an overly optimistic picture. ${ }^{11}$ And, a large UK study reported reductions in CLABSI comparable to those 
in Michigan, yet did not produce an improvement over and above the secular trend. ${ }^{12}$ Moreover, preICU infections declined in parallel with ICU-acquired infections, suggesting effects other than the bundle. As noted in the companion editorial, ${ }^{8}$ outcome ascertainment for CLABSI (and CAUTI) depends on clinical behaviours. Many clinicians reflexively 'pan culture' patients when their clinical status worsens. ${ }^{13}$ This habit produces numerous false positive results. Curbing this habit represents an improvement. Yet, reducing the frequency of cultures will reduce CLABSI and CAUTI even without other specific improvements.

\section{CEILING EFFECTS VERSUS SURVEILLANCE BIAS}

Less frequent culturing of blood and urine would give rise to surveillance bias as a cause for some of the reductions in CLABSI and CAUTI reported in past studies, as well as the apparent secular improvements invoked as the basis for ceiling effects in the study by Meddings et al. ${ }^{1}$ Various studies have identified important differences in the detection, documentation and reporting of CLABSIs between centres. ${ }^{14-16}$ Comparable data do not exist for CAUTI. But, the previous AHRQ On the CUSP: Stop CAUTI project ${ }^{3}$ showed essentially no improvement for ICUs, with a CAUTI rate of 2.50 per 1000 catheter days for ICUs at the end of the evaluation. Yet ICUs in the current programme started with a rate of 1.25 per 1000 urinary catheter days. ${ }^{1}$ This CAUTI rate is $50 \%$ lower than the ICUs at the end of the previous project, and it surpasses the rate observed on units outside the ICU, where the intervention succeeded. If ICUs participating in a major national collaborative $e^{3}$ could not reduce their CAUTI rate, it seems unlikely that non-participating ICUs would cut their rate by $50 \%$ over just a few years. If secular trends produced such substantial improvements, we would have no need of improvement collaboratives.

What might cause decreased clinical surveillance for CLABSI and CAUTI (ie, less frequent testing with blood and urine cultures)? Widespread attention to Choosing Wisely over the past 8 years ${ }^{1718}$ has generally included a focus on reducing unnecessary laboratory tests. ${ }^{19-21}$ Unnecessary urine cultures have received specific attention. ${ }^{22-25}$ One initiative specifically highlighted the role that 'stewardship of culturing' played in reducing CAUTI. ${ }^{23}$ None of the five participating ICUs in that initiative showed significant changes in the monthly urine culturing rate during the preintervention period. Just 1 month into the intervention, urine culturing rates decreased significantly $(p=0.001)$ across all five units, with significant downward trends continuing throughout the postintervention period. ${ }^{23}$

\section{HAVE WE FOCUSED TOO MUCH ON NARROW HEALTHCARE-ACQUIRED COMPLICATIONS?}

I do not suggest that all past reductions in CLABSI or CAUTI entirely reflect surveillance bias. Bundles targeting these outcomes consist of specific elements with well-established benefits. The use of chlorhexidine for skin disinfection on its own delivers a substantial reduction in CLABSI. ${ }^{26}{ }^{27}$ Greater use of this and other bundle elements ought to reduce the rate at which CLABSI occurs. The same holds true for the CAUTI bundle, which consists of elements that almost certainly reduce the rate at which this complication occurs. So, advocates of these bundles will argue that the problem consists primarily of an implementation challenge.

The question, though, 20 years into the patient safety movement, is how far to pursue such implementation challenges. CLABSI and CAUTI constitute very narrow targets. We still have other healthcare-acquired infections to target and many non-infectious healthcareacquired complications (fall-related injuries, pressure ulcers, venous thromboembolism, delirium, etc), numerous different types of preventable adverse drug events, complications after surgery and other invasive procedures, and harms from misdiagnosis. ${ }^{28-30}$ Are we really going to continue to pursue a 'whack-a-mole' approach, trying to reduce so many specific harms? It seems like a futile endeavour given that CLABSI and CAUTI, both of which have received considerable attention over 10-15 years, have produced mixed or unclear successes. Moreover, healthcare professionals complain of change fatigue from numerous improvement initiatives and exhibit symptoms of burn-out in general. $^{31}$

I raise this question recognising that I and others initially advocated for greater attention to precisely these sorts of patient safety targets. ${ }^{32}$ Following publication of To Err is Human, ${ }^{33}$ AHRQ commissioned an evidence report ranking over 80 specific patient practices on the basis of strength of evidence, scope of the specific safety problem, implementation issues and the possibility for unintended consequences. ${ }^{34}$ Several prominent experts expressed dismay over the prominence of interventions to prevent venous thromboembolism and various other harms of hospitalisation. ${ }^{35}$ They had expected recommendations to emulate safety practices from industries such as aviation and nuclear power, including a focus on incident investigation ${ }^{36} 37$ and application of principles from human factors engineering. ${ }^{38} 39$ Expressing surprise over the mundane clinical targets of the recommended interventions, they wrote: 'While the safety of central venous catheter use is important, it would not be near the top of the list of challenges for hospital safety officers or near the top of the list of causes of patient harm. Similarly, preventing ventilator-associated pneumonia... has limited application'. ${ }^{35}$

Nonetheless, the ensuing years have seen a proliferation of efforts to prevent CLABSI, CAUTI, venous thromboembolism, ventilator-associated pneumonia, falls, pressure ulcers, surgical site infections and so on. The experience with CLABSI and CAUTI makes me 
wonder if this focus on narrow safety targets achieves too little benefit and comes at too high cost-in personnel time and staff morale.

\section{FROM CONFUSING COMPLEXITY TO PROFOUND SIMPLICITY: CULTURAL CHANGE AS AN END IN ITSELF}

The organisational theorist Karl Wieck has written that 'understanding progresses through three stages: superficial simplicity, confused complexity, followed by profound simplicity'. ${ }^{40}$ The superficial simplicity in patient safety consisted of calls to simply emulate other high-risk industries. Initial critiques, including from me, ${ }^{41}$ highlighted the need for more evidence about specific interventions and how to implement them. Others pointed out that calls to emulate the benefits of checklists reflected a superficial understanding of how other industries derived benefit from such tools. ${ }^{42} 43$ They did not use checklists as socioadaptive interventions. They spent decades investing directly in culture change and team training, as well as designing better systems.

In patient safety, Weick's 'confusing complexity' describes the seemingly endless list of specific harms to target, as well as attempts to combine seemingly straightforward interventions such as bundles and checklists with more complex behavioural and cultural changes. In my experience, a checklist really is just a checklist. When I go to the market, I want to quickly glance at a list of items so as not to forget anything, not engage in a shared mental model with anyone. Granted, surgery constitutes a more complex, teambased activity than my simple, solo trip to the market. Still, if achieving the desired impact of a checklist or bundle requires major changes to how we communicate and behave, we need to target those desired changes, not just relegate them to the 'implementation plan'. Real improvements in teamwork and communication increasingly seem like worthwhile goals in themselves, not just the socioadaptive means to the end of implementing checklists and bundles.

We have reasons to focus on removal of catheters, especially urinary ones. Patients often find them uncomfortable and they reduce mobility. ${ }^{44}$ But the evidence for interventions to reduce narrow targets such as CAUTI and CLABSI remains mixed, and efforts to implement them produce change fatigue. We need ways to improve care for patients which also improve staff morale. $^{45} 46$

The 20 years since To Err is Human ${ }^{33}$ have produced too little progress with narrow targets such as CLABSI and CAUTI. Even when checklist interventions include strategies for supporting cultural change, such as CUSP, they do not necessarily work. ${ }^{47}$ Yet interventions focusing on teamwork and cultural change, where a checklist constitutes one component, not the main event, have reported important improvements in patient outcomes. ${ }^{48}$ This increasingly seems to me the 'profound simplicity' ${ }^{40}$ we need to accept. How clinicians communicate and interact-with each other and with patients-constitute the main events. Focusing more intensely on these activities than we have to date now seems to hold more promise for patient safety and staff morale than does the Sisyphean task of developing, implementing and sustaining interventions targeting one narrow safety problem after another.

Acknowledgements The author gratefully acknowledges feedback on earlier drafts of this manuscript from Dr. Julian Bion and Dr. Michael Klompas, as well as several of the editors at BMJ Quality and Safety.

Funding The authors have not declared a specific grant for this research from any funding agency in the public, commercial or not-for-profit sectors.

Competing interests KGS is one of the co-Editor-in-Chief at BMJ Quality and Safety.

Patient consent for publication Not required.

Provenance and peer review Commissioned; internally peer reviewed.

\section{REFERENCES}

1 Meddings J, Greene MT, Ratz D, et al. Multistate programme to reduce catheter-associated infections in intensive care units with elevated infection rates. BMJ Qual Saf 2020;29:418-29.

2 Berenholtz SM, Lubomski LH, Weeks K, et al. Eliminating central line-associated bloodstream infections: a national patient safety imperative. Infect Control Hosp Epidemiol 2014;35:56-62.

3 Saint S, Greene MT, Krein SL, et al. A program to prevent catheter-associated urinary tract infection in acute care. $\mathrm{N}$ Engl J Med 2016;374:2111-9.

4 Dixon-Woods M, Bosk CL, Aveling EL, et al. Explaining Michigan: developing an ex post theory of a quality improvement program. Milbank Q 2011;89:167-205.

5 Bosk CL, Dixon-Woods M, Goeschel CA, et al. Reality check for checklists. Lancet 2009;374:444-5.

6 Pronovost P, Needham D, Berenholtz S, et al. An intervention to decrease catheter-related bloodstream infections in the ICU. N Engl J Med 2006;355:2725-32.

7 Pronovost PJ. Navigating adaptive challenges in quality improvement. BMJ Qual Saf 2011;20:560-3.

8 Chopra V, Shojania KG. Recipes for checklists and bundles: one part active ingredient, two parts measurement. BMJ Qual Saf 2013;22:93-6.

9 Meddings J, Reichert H, Greene MT, et al. Evaluation of the association between hospital survey on patient safety culture (HSOPS) measures and catheter-associated infections: results of two national collaboratives. BMJ Qual Saf 2017;26:226-35.

10 Marang-van de Mheen PJ, van Bodegom-Vos L. Meta-analysis of the central line bundle for preventing catheter-related infections: a case study in appraising the evidence in quality improvement. BMJ Qual Saf 2016;25:118-29.

11 Daley MR. Catheter-related bloodstream infections. N Engl J Med 2007;356:1267-8.

12 Bion J, Richardson A, Hibbert P, et al. 'Matching Michigan': a 2-year stepped interventional programme to minimise central venous catheter-blood stream infections in intensive care units in England. BMJ Qual Saf 2013;22:110-23.

13 Vaughn VM, Chopra V. Revisiting the panculture. BMJ Qual Saf 2017;26:236-9. 
14 Niedner MF, 2008 National Association of Children's Hospitals and Related Institutions Pediatric Intensive Care Unit Patient Care FOCUS Group. The harder you look, the more you find: catheter-associated bloodstream infection surveillance variability. Am J Infect Control 2010;38:585-95.

15 Aswani MS, Reagan J, Jin L, et al. Variation in public reporting of central line-associated bloodstream infections by state. Am J Med Qual 2011;26:387-95.

16 Dixon-Woods M, Leslie M, Bion J, et al. What counts? An ethnographic study of infection data reported to a patient safety program. Milbank Q 2012;90:548-91.

17 Cassel CK, Guest JA. Choosing wisely: helping physicians and patients make smart decisions about their care. JAMA 2012;307:1801-2.

18 Levinson W, Kallewaard M, Bhatia RS, et al. 'Choosing wisely': a growing international campaign. BMJ Qual Saf 2015;24:167-74.

19 Leis B, Frost A, Bryce R, et al. Altering standard admission order sets to promote clinical laboratory stewardship: a cohort quality improvement study. BMJ Qual Saf 2019;28:846-52.

20 Strauss R, Cressman A, Cheung M, et al. Major reductions in unnecessary aspartate aminotransferase and blood urea nitrogen tests with a quality improvement initiative. BMJ Qual Saf 2019;28:809-16.

21 Fang DZ, Sran G, Gessner D, et al. Cost and turn-around time display decreases inpatient ordering of reference laboratory tests: a time series. BMJ Qual Saf 2014;23:994-1000.

22 Epstein L, Edwards JR, Halpin AL, et al. Evaluation of a novel intervention to reduce unnecessary urine cultures in intensive care units at a tertiary care hospital in Maryland, 2011-2014. Infect Control Hosp Epidemiol 2016;37:606-9.

23 Mullin KM, Kovacs CS, Fatica C, et al. A Multifaceted Approach to Reduction of Catheter-Associated Urinary Tract Infections in the Intensive Care Unit With an Emphasis on "Stewardship of Culturing". Infect Control Hosp Epidemiol 2017;38:186-8.

24 Munigala S, Jackups RR, Poirier RF, et al. Impact of order set design on urine culturing practices at an academic medical centre emergency department. BMJ Qual Saf 2018;27:587-92.

25 Stagg A, Lutz H, Kirpalaney S, et al. Impact of two-step urine culture ordering in the emergency department: a time series analysis. BMJ Qual Saf 2018;27:140-7.

26 Chaiyakunapruk N, Veenstra DL, Lipsky BA, et al. Chlorhexidine compared with povidone-iodine solution for vascular catheter-site care: a meta-analysis. Ann Intern Med 2002;136:792-801.

27 Mimoz O, Lucet J-C, Kerforne T, et al. Skin antisepsis with chlorhexidine-alcohol versus povidone iodine-alcohol, with and without skin scrubbing, for prevention of intravascularcatheter-related infection (clean): an open-label, multicentre, randomised, controlled, two-by-two factorial trial. Lancet 2015;386:2069-77.

28 Singh H, Schiff GD, Graber ML, et al. The global burden of diagnostic errors in primary care. BMJ Qual Saf 2017;26:484-94.

29 Graber ML. The incidence of diagnostic error in medicine. BMJ Qual Saf 2013;22:ii21-7.
30 Shojania KG, Burton EC, McDonald KM, et al. Changes in rates of autopsy-detected diagnostic errors over time: a systematic review. JAMA 2003;289:2849-56.

31 Dzau VJ, Kirch DG, Nasca TJ. To Care Is Human Collectively Confronting the Clinician-Burnout Crisis. N Engl J Med 2018;378:312-4.

32 Shojania KG, Duncan BW, McDonald KM, et al. Safe but sound: patient safety meets evidence-based medicine. JAMA 2002;288:508-13.

33 Kohn LT, Corrigan J, Donaldson MS. To err is human: building a safer health system. Washington, D.C: National Academy Press, 2000.

34 Shojania KG, Duncan BW, McDonald KM, et al. Making health care safer: a critical analysis of patient safety practices. Evid Rep Technol Assess 2001:1-668.

35 Leape LL, Berwick DM, Bates DW. What practices will most improve safety? Evidence-based medicine meets patient safety. JAMA 2002;288:501-7.

36 Mitchell I, Schuster A, Smith K, et al. Patient safety incident reporting: a qualitative study of thoughts and perceptions of experts 15 years after 'To Err is Human'. BMJ Qual Saf 2016;25:92-9.

37 Travaglia JF, Hughes C, Braithwaite J. Learning from disasters to improve patient safety: applying the generic disaster pathway to health system errors. BMJ Qual Saf 2011;20:1-8.

38 Gurses AP, Kim G, Martinez EA, et al. Identifying and categorising patient safety hazards in cardiovascular operating rooms using an interdisciplinary approach: a multisite study. BMJ Qual Saf 2012;21:810-8.

39 Gurses AP, Ozok AA, Pronovost PJ. Time to accelerate integration of human factors and ergonomics in patient safety. BMJ Qual Saf 2012;21:347-51.

40 Weick KE. Perspective construction in organizational behavior. Annual Review of Organizational Psychology and Organizational Behavior 2017;4:1-17.

41 Auerbach AD, Landefeld CS, Shojania KG. The tension between needing to improve care and knowing how to do it. $N$ Engl J Med 2007;357:608-13.

42 Catchpole K, Russ S. The problem with checklists. BMJ Qual Saf 2015;24:545-9.

43 Clay-Williams R, Colligan L. Back to basics: checklists in aviation and healthcare. BMJ Qual Saf 2015;24:428-31.

44 Saint S, Lipsky BA, Goold SD. Indwelling urinary catheters: a one-point restraint? Ann Intern Med 2002;137:125-7.

45 Smaggus A. Safety-I, Safety-II and burnout: how complexity science can help clinician wellness. BMJ Qual Saf 2019;28:667-71.

46 Sikka R, Morath JM, Leape L. The quadruple aim: care, health, cost and meaning in work. BMJ Qual Saf 2015;24:608-10.

47 Reames BN, Krell RW, Campbell DA, et al. A checklist-based intervention to improve surgical outcomes in Michigan: evaluation of the keystone surgery program. JAMA Surg 2015;150:208-15.

48 Neily J, Mills PD, Young-Xu Y, et al. Association between implementation of a medical team training program and surgical mortality. JAMA 2010;304:1693-700. 